\title{
SOME ASPECTS OF TOPOLOGY AND KINEMATICS OF A 3DOF TRANSLATIONAL PARALLEL MECHANISM
}

\author{
J. BAŁCHANOWSKI \\ Faculty of Mechanical Engineering \\ Wrocław University of Technology \\ 50-371 Wrocław, Łukasiewicza 7/9, POLAND \\ E-mail: Jacek.Balchanowski@pwr.wroc.pl
}

\begin{abstract}
The paper presents elements of the topology, geometry and the kinematic analysis of a translational parallel mechanism with three degrees of freedom. In such mechanisms the selection of a proper structure and geometry ensures that the driven link maintains a fixed orientation relative to the base. The method of determination of the configuration of mechanisms using contour vector notation was elaborated in the paper. The equations for the analysis of the direct and inverse kinematics task are determined. An analytical procedure for determining the system's singular positions is presented and illustrated with examples.
\end{abstract}

Key words: the parallel mechanisms, topology, direct and inverse kinematic, singularities.

\section{Introduction}

The term parallel mechanisms commonly refers to kinematic systems whose driven link is connected with the base through several independent kinematic chains. The parallel mechanism find an ever wider application as: motion simulators, specialized manipulators and numerical machine tools (Tsai, 1999; Merlet, 2000).

The research on parallel mechanisms was conducted in the Mechanical Engineering Faculty at Wrocław University of Technology (Bałchanowski et al., 2001). In the study, a new general method for the type synthesis of parallel mechanisms was worked out. Using this method, systematic catalogues of structures of parallel mechanisms for a specific mobility were developed. In the group of systems with 3 DOF more than 50 structures mechanisms were obtained. Some structures were already known in the literature and some were new solutions.

The paper deals with the geometry and direct and inverse kinematics of a 3DOF translational parallel mechanism with linear drives denoted in Bałchanowski et al. (2001), Bałchanowski and Gronowicz (2001) as MR6U3T (Fig.1), wider known as Tsai 3-UPU (Tsai, 1996) and later in the paper denoted MT-3-UPU. This mechanism and its generalizations were discussed with respect to kinematic properties especially focused on the possibility of generating pure translational motion in (e.g., Tsai, 1996; Di Gregorio and Parenti-Castelli, 1999; Kim and Chung, 2003; Bałchanowski, 2008; Walter and Husty, 2011; Chebii Ahmeg et al., 2013). The method of determination of mechanism configurations using contour vector notation was elaborated in the paper.

One of the disadvantages of parallel mechanism is the possibility of singular configurations occurring in the workspace. A mechanism, being in a singular configuration, changes the properties - the values of some parameters (forces, velocities and accelerations) become indeterminate or approach infinity. The determination of these configurations is a challenging and complex problem and has motivated many researchers to study various techniques and methods in this area to describe these phenomena (e.g., Wolf $e t$ al., 2002; Guanfeng Liu et al., 2003; Gogu, 2008; Peng Binbin et al., 2011; Bałchanowski, 2014). In the work an analytical method of determination of singular configurations for direct and inverse task of 
kinematics was worked out. The method is based on analysing the equations of velocity. At singular configurations, the determinant of the mechanism velocities matrix becomes zero. Using this method the singular configurations of the mechanism under analysis were determined and illustrated.

\section{Design and configuration of the MT-3-UPU parallel mechanism}

The subject of analysis is a translational parallel mechanism with three linear drives (Fig.1). In such mechanisms the selection of a proper structure and geometry ensures that the driven link maintains a fixed orientation relative to the base, i.e., it can move only translationally (Tsai, 1996; Di Gregorio and ParentiCastelli, 1999; Bałchanowski, 2001; 2014). The analyzed mechanism consists of three branches connecting the driven link $b$ (platform) with base 0 (Fig.1) using 6 universal joints and 3 prismatic joints. The mechanism has three degrees of freedom whereby three kinematic excitations (drives) need to be employed.

a)

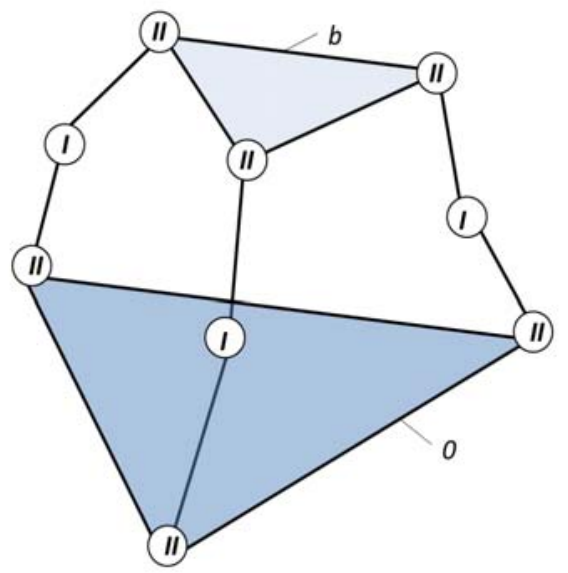

b)

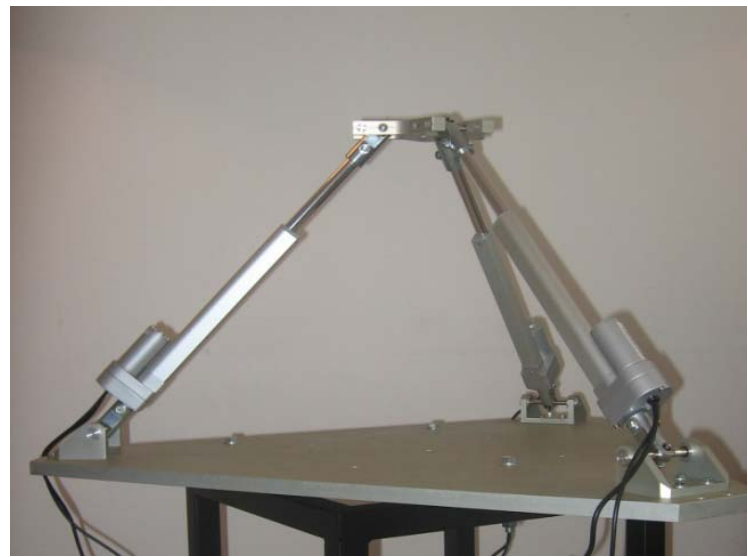

Fig.1. The MT-3-UPU parallel mechanism: a) topological scheme, b) general view of real system.

Linear excitations $q_{1}, q_{2}$ and $q_{3}$ describing the displacements in prismatic joints (Figs 2 and 3) were adopted as independent variables.

For each system with a determined motion one can write the equations of constraints in the form (Gronowicz, 2003; Bałchanowski, 2014)

$$
\boldsymbol{\Phi}=\left[\begin{array}{c}
\boldsymbol{\Phi}^{P}(\boldsymbol{q}) \\
\boldsymbol{\Phi}^{C}(\boldsymbol{q}, t)
\end{array}\right]=0
$$

where: $\boldsymbol{\Phi}^{P}$ - a matrix of equations of the constraints resulting from the connection of the links in joints,

$\boldsymbol{\Phi}^{C}$ - a matrix of the constraints describing the kinematic excitations,

$\boldsymbol{q}$ - a matrix of the generalized coordinates describing the system configurations.

The MT-3-UPU mechanism equations of constraints (configuration) were described using vector notation. And so the kinematic chain was replaced by a proper vector chain. In the considered case, three closed contours, each for a different mechanism branch, were created. The closing vector was the vector $\boldsymbol{r}_{\boldsymbol{M}}$ describing the position of the platform $b$ (Fig.3).

The global coordinate system $x_{\mathrm{o}} y_{o} z_{o}$ and local coordinate systems $x_{i} y_{i} z_{i}$ were defined on the links of the mechanism. Dimensions $r_{d}, r_{g}$ and angles $\gamma_{1}, \gamma_{2}, \gamma_{3}$ specify the arrangement (Fig.2) of the kinematic pairs $A, B, C$ on base 0 and pairs $P, R, S$ on the driven link $b$ (the platform). Assuming the above coordinate systems, the following vector equation was written for each branch 


$$
\begin{aligned}
& \boldsymbol{r}_{A}+\boldsymbol{r}_{q 1}=\boldsymbol{r}_{M}+\boldsymbol{r}_{p}, \\
& \boldsymbol{r}_{B}+\boldsymbol{r}_{q 2}=\boldsymbol{r}_{M}+\boldsymbol{r}_{S}, \\
& \boldsymbol{r}_{C}+\boldsymbol{r}_{q 3}=\boldsymbol{r}_{M}+\boldsymbol{r}_{R} .
\end{aligned}
$$

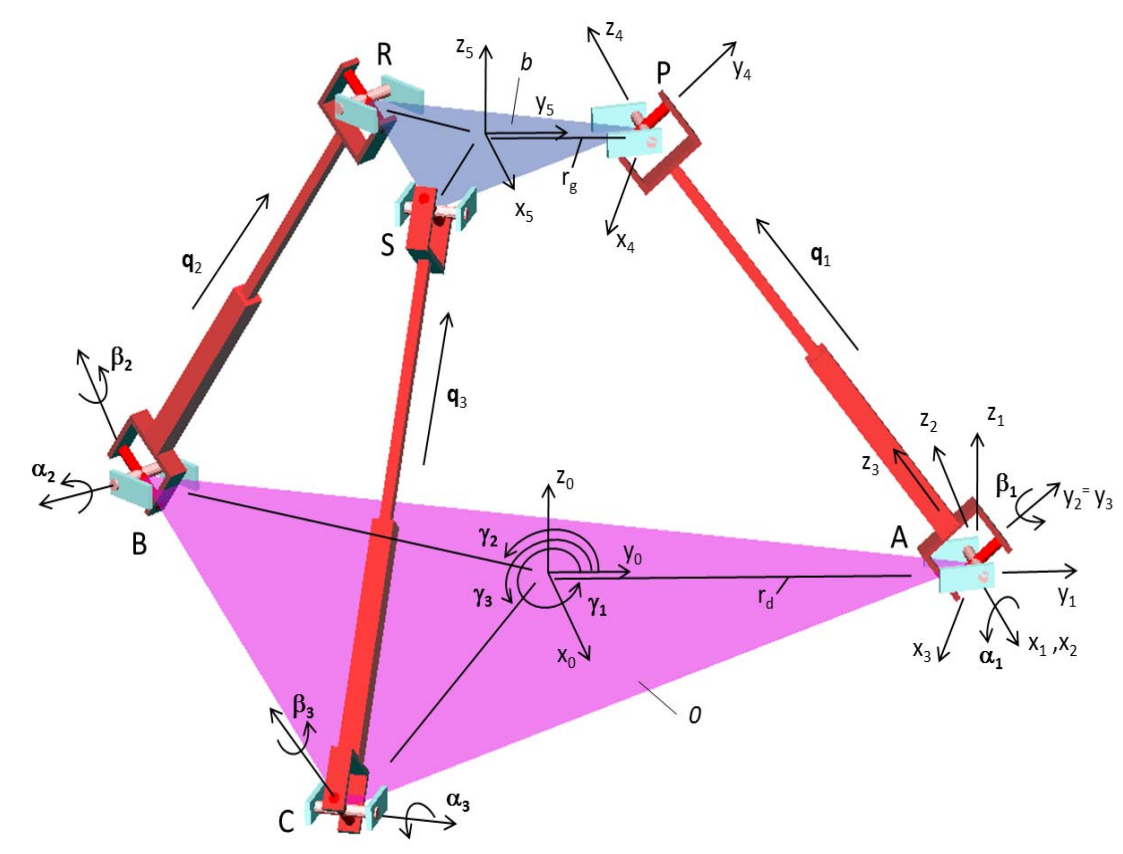

Fig.2. Kinematic model of MT-3-UPU parallel mechanism.

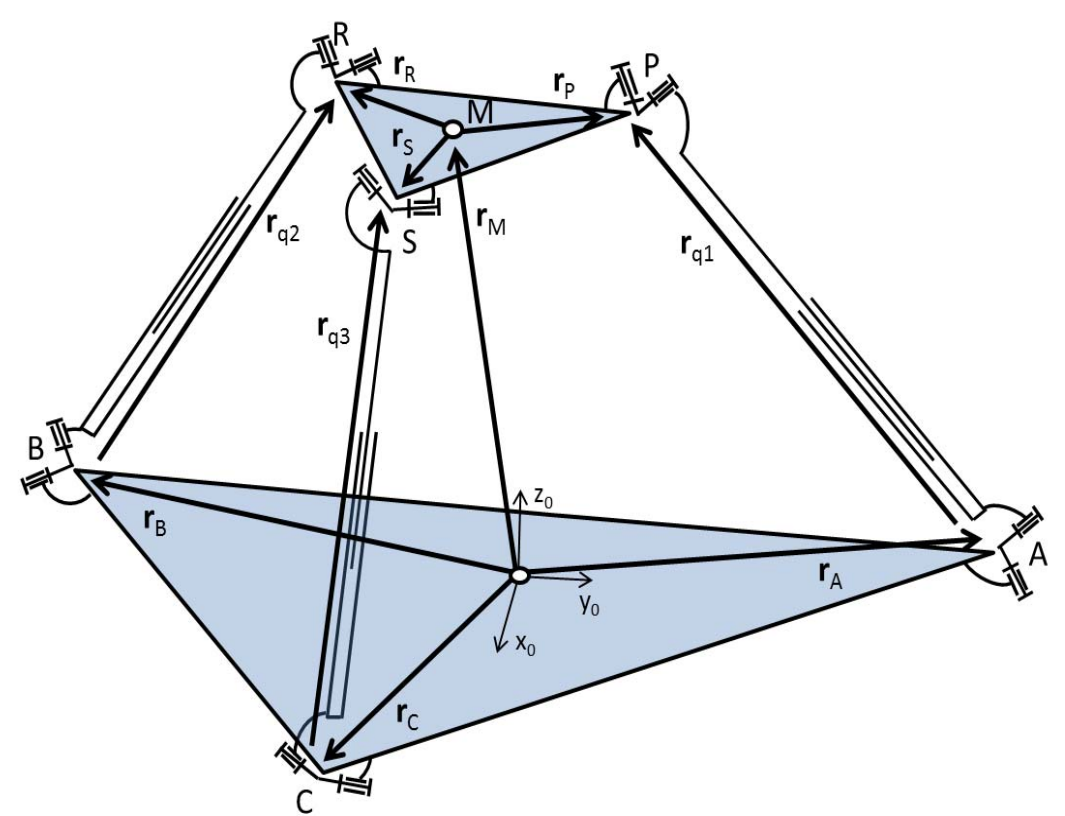

Fig.3. Vector contours against MT-3-UPU parallel mechanism. 
The left side of Eq.(2.2), describing branch $A P$ was determined by introducing the transition matrices

$$
\boldsymbol{r}_{A}+\boldsymbol{r}_{q 1}=A_{01} A_{11} A_{12} A_{23} A_{34}{ }^{4} \boldsymbol{r}_{p}
$$

where: ${ }^{4} \boldsymbol{r}_{p}=\left[\begin{array}{llll}0 & 0 & 0 & 1\end{array}\right]^{T}$ - a vector describing the position of point $P$ on the link in the system 4 ,

$\alpha_{1}, \beta_{l}$ - angles of rotation in the universal joint $A$,

$A_{i j}$ - matrices of transformation: from $i$ to $j$ system

$$
\begin{array}{rlrl}
\boldsymbol{A}_{01} & =\left[\begin{array}{cccc}
\cos \gamma_{1} & -\sin \gamma_{1} & 0 & 0 \\
\sin \gamma_{1} & \cos \gamma_{1} & 0 & 0 \\
0 & 0 & 1 & 0 \\
0 & 0 & 0 & 1
\end{array}\right], & \boldsymbol{A}_{11}=\left[\begin{array}{llll}
1 & 0 & 0 & 0 \\
0 & 1 & 0 & r_{d} \\
0 & 0 & 1 & 0 \\
0 & 0 & 0 & 1
\end{array}\right], \\
\boldsymbol{A}_{23} & =\left[\begin{array}{cccc}
\cos \beta_{1} & 0 & \sin \beta_{1} & 0 \\
0 & 1 & 0 & 0 \\
-\sin \beta_{1} & 0 & \cos \beta_{1} & 0 \\
0 & 0 & 0 & 1
\end{array}\right], & \boldsymbol{A}_{12}=\left[\begin{array}{cccc}
1 & 0 & 0 & 0 \\
0 & \cos \alpha_{1} & -\sin \alpha_{1} & 0 \\
0 & \sin \alpha_{1} & \cos \alpha_{1} & 0 \\
0 & 0 & 0 & 1
\end{array}\right], \\
& \boldsymbol{A}_{34}=\left[\begin{array}{llll}
1 & 0 & 0 & 0 \\
0 & 1 & 0 & 0 \\
0 & 0 & 0 & q_{1} \\
0 & 0 & 0 & 1
\end{array}\right] .
\end{array}
$$

The vector $\boldsymbol{r}_{M}$ describes the position of point $M$ on the platform in the global system (Fig.3)

$$
r_{M}=\left[\begin{array}{llll}
x_{M} & y_{M} & z_{M} & 1
\end{array}\right]^{T} .
$$

The vector $\boldsymbol{r}_{p}$ on the platform $b$ one can presented in the global system 0 using the formula (Fig.3)

$$
\boldsymbol{r}_{P}=A_{50}{ }^{5} \boldsymbol{r}_{p}
$$

where ${ }^{5} r_{p}=\left[\begin{array}{lll}0 & r_{g} & 0\end{array}\right]^{T}-$ the vector describes point $P$ in system 5 ,

$$
A_{50}=\left[\begin{array}{cccc}
\cos \gamma_{1} & -\sin \gamma_{1} & 0 & 0 \\
\sin \gamma_{1} & \cos \gamma_{1} & 0 & 0 \\
0 & 0 & 1 & 0 \\
0 & 0 & 0 & 1
\end{array}\right]
$$

After components of Eq.(2.5), Eq.(2.6) and Eq.(2.7) are determined and elementary transformations performed Eq.(2.1) assumes this form

$$
\Phi_{I}^{p}=\left[\begin{array}{l}
r_{g} \sin \gamma_{1}-r_{d} \sin \gamma_{1}-x_{M}+q_{1} \cos \gamma_{1} \sin \beta_{1}+q_{1} \sin \gamma_{1} \cos \beta_{1} \sin \alpha_{1} \\
r_{d} \cos \gamma_{1}-y_{M}-r_{g} \cos \gamma_{1}+q_{1} \sin \gamma_{1} \sin \beta_{1}-q_{1} \cos \gamma_{1} \cos \beta_{1} \sin \alpha_{1} \\
q_{1} \cos \alpha_{1} \cos \beta_{1}-z_{M}
\end{array}\right]=\boldsymbol{0} .
$$

Transforming in a similar way Eq.(2.3) and Eq.(2.4) for branches $B R$ and $C S$ (Figs 3 and 4) and performing transformations, the following constraints equations $\Phi^{P}{ }_{i}$ for BR $(i=2)$ and for CS $(i=3)$ were obtained 


$$
\boldsymbol{\Phi}_{i}^{p}=\left[\begin{array}{l}
r_{g} \sin \gamma_{i}-r_{d} \sin \gamma_{i}-x_{M}+q_{i} \cos \gamma_{i} \sin \beta_{i}+q_{i} \sin \gamma_{i} \cos \beta_{i} \sin \alpha_{i} \\
r_{d} \cos \gamma_{i}-y_{M}-r_{g} \cos \gamma_{i}+q_{i} \sin \gamma_{i} \sin \beta_{i}-q_{i} \cos \gamma_{i} \cos \beta_{i} \sin \alpha_{i} \\
q_{i} \cos \alpha_{i} \cos \beta_{i}-z_{M}
\end{array}\right]=\mathbf{0}
$$

where $\alpha_{2}, \beta_{2}, \alpha_{3}, \beta_{3}$ - angles of rotation in the universal joints $B$ and $C$.

The matrix of constraints $\Phi^{P}$, in accordance with Eq.(1.1), for the MT-3-UPU parallel mechanism assumes the form

$$
\Phi^{P}=\left[\Phi_{1}^{P} \Phi_{2}^{P} \Phi_{3}^{P}\right]^{T}=0
$$

\subsection{Direct kinematic task of the mechanism MT-3-UPU}

The direct kinematics task consists in determining the position of driven link $b$, defined by the vector $\boldsymbol{r}_{M}$, on the basis of prescribed linear kinematic excitations $q_{1}, q_{2}, q_{3}$ (Figs 2 and 3). Hence, according to Eq.(2.1), the constraints column matrix $\Phi^{c}$ describing the kinematic excitations (drives) for the direct problem assumes the form

$$
\Phi^{C}=\Phi_{d i r}^{c}=\left[q_{1}(t), q_{2}(t), q_{3}(t)\right]^{T}
$$

and unknown constraints vector $\boldsymbol{q}$ has the form

$$
\boldsymbol{q}=\boldsymbol{q}_{d i r}=\left[\alpha_{1}, \beta_{1}, x_{M}, \alpha_{2}, \beta_{2}, y_{M}, \alpha_{3}, \beta_{3}, z_{M}\right]^{T} \text {. }
$$

By solving Eq.(2.10), the system of nine nonlinear equations $\Phi^{P}$ for the given $\Phi_{\text {dir }}^{C}$, one gets unknown configuration $\boldsymbol{q}_{d i}$ of the mechanism, in the form of the position of platform $b$ and the angles of rotation in the joints.

In the direct kinematics problem the velocity equation, being the basis for determining singular positions, was obtained by differentiating Eq.(2.10) over time and has this form

$$
\boldsymbol{A}_{d i r} \dot{\boldsymbol{q}}_{d i r}=\boldsymbol{B}_{d i r} \dot{\Phi}_{d i r}^{c}
$$

where

$$
\begin{aligned}
\dot{\boldsymbol{q}}_{d i r} & =\left[\dot{\alpha}_{1}, \dot{\beta}_{1}, \dot{x}_{M}, \dot{\alpha}_{2}, \dot{\beta}_{2}, \dot{y}_{M}, \dot{\alpha}_{3}, \dot{\beta}_{3}, \dot{z}_{M}\right]^{T}, \\
\dot{\boldsymbol{\Phi}}_{d i r}^{c} & =\left[\dot{q}_{1}, \dot{q}_{2}, \dot{q}_{3}\right]^{T}, \\
\boldsymbol{B}_{d i r} & =\left[\begin{array}{ccccccccc}
f_{1}^{1} & 0 & 0 & f_{1}^{2} & 0 & 0 & f_{1}^{3} & 0 & 0 \\
0 & f_{2}^{l} & 0 & 0 & f_{2}^{2} & 0 & 0 & f_{2}^{3} & 0 \\
0 & 0 & f_{3}^{1} & 0 & 0 & f_{3}^{2} & 0 & 0 & f_{3}^{3}
\end{array}\right]^{T},
\end{aligned}
$$


and for

$$
\begin{aligned}
& j=1,2,3, \\
& f_{1}^{j}=-\left(\cos \gamma_{j} \sin \beta_{j}+\sin \gamma_{j} \cos \beta_{j} \sin \alpha_{j}\right), \\
& f_{2}^{j}=-\left(\sin \gamma_{j} \sin \beta_{j}-\cos \gamma_{j} \cos \beta_{j} \sin \alpha_{j}\right), \\
& f_{3}^{j}=-\left(\cos \alpha_{j} \cos \beta_{j}\right) .
\end{aligned}
$$

The matrix $\boldsymbol{A}_{\text {dir }}$ for direct kinematic task has the form

$$
\boldsymbol{A}_{\text {dir }}=\left[\begin{array}{ccccccccc}
a_{11}^{1} & a_{12}^{1} & -1 & 0 & 0 & 0 & 0 & 0 & 0 \\
a_{21}^{1} & a_{22}^{1} & 0 & 0 & 0 & -1 & 0 & 0 & 0 \\
a_{31}^{1} & a_{32}^{1} & 0 & 0 & 0 & 0 & 0 & 0 & -1 \\
0 & 0 & -1 & a_{11}^{2} & a_{12}^{2} & 0 & 0 & 0 & 0 \\
0 & 0 & 0 & a_{21}^{2} & a_{22}^{2} & -1 & 0 & 0 & 0 \\
0 & 0 & 0 & a_{31}^{2} & a_{32}^{2} & 0 & 0 & 0 & -1 \\
0 & 0 & -1 & 0 & 0 & 0 & a_{11}^{3} & a_{12}^{3} & 0 \\
0 & 0 & 0 & 0 & 0 & -1 & a_{21}^{3} & a_{22}^{3} & 0 \\
0 & 0 & 0 & 0 & 0 & 0 & a_{31}^{3} & a_{32}^{3} & -1
\end{array}\right]
$$

where for $\quad j=1,2,3$,

$$
\begin{array}{ll}
a_{11}^{j}=q_{j} \sin \gamma_{j} \cos \alpha_{j} \cos \beta_{j}, & a_{22}^{j}=q_{j} \sin \gamma_{j} \cos \beta_{j}+q_{j} \cos \gamma_{j} \sin \alpha_{j} \sin \beta_{j}, \\
a_{12}^{j}=q_{j} \cos \gamma_{j} \cos \beta_{j}-q_{j} \sin \gamma_{j} \sin \alpha_{j} \sin \beta_{j}, & a_{31}^{j}=-q_{j} \cos \beta_{j} \sin \alpha_{j}, \\
a_{21}^{j}=-q_{j} \cos \gamma_{j} \cos \alpha_{j} \cos \beta_{j}, & a_{32}^{j}=-q_{j} \cos \alpha_{j} \sin \beta_{j} .
\end{array}
$$

\subsection{Inverse kinematic task of the MT-3-UPU mechanism}

The inverse kinematics task consists in determining the kinematic excitations $q_{1}, q_{2}, q_{3}$ on the basis of the known position of driven link b (platform), defined by vector $\boldsymbol{r}_{M}$. The constraints column matrix $\boldsymbol{\Phi}^{c}$ of the kinematic excitations (drives), according to Eq.(2.1), for the inverse kinematic problem has the form

$$
\Phi^{C}=\Phi_{i n v}^{c}=\left[x_{M}(t), y_{M}(t), z_{M}(t)\right]^{T},
$$

and unknown constraints vector $\boldsymbol{q}$ has the form 


$$
\boldsymbol{q}=\boldsymbol{q}_{\text {inv }}=\left[\alpha_{1}, \beta_{1}, q_{1}, \alpha_{2}, \beta_{2}, q_{2}, \alpha_{3}, \beta_{3}, q_{3}\right]^{T}
$$

Using configuration $\Phi^{P}$ Eq.(2.10) one can build a system of equations for given $\Phi_{i n v}^{c}$ and by solving it for $\boldsymbol{q}_{i n v}$ one can solve the inverse kinematics problem.

One can get equations of velocities after differentiation of the constraints Eq.(2.10) and making the necessary transformations in the form

$$
\boldsymbol{A}_{i n v} \dot{\boldsymbol{q}}_{i n v}=\boldsymbol{B}_{i n v} \dot{\Phi}_{i n v}^{c}
$$

where

$$
\begin{aligned}
& \dot{\boldsymbol{q}}_{i n v}=\left[\dot{\alpha}_{1}, \dot{\beta}_{1}, \dot{q}_{1}, \dot{\alpha}_{2}, \dot{\beta}_{2}, \dot{q}_{2}, \dot{\alpha}_{3}, \dot{\beta}_{3}, \dot{q}_{3}\right]^{T}, \\
& \dot{\boldsymbol{\Phi}}_{i n v}^{c}=\left[\dot{x}_{M}, \dot{y}_{M}, \dot{z}_{M}\right]^{T}, \\
& \boldsymbol{B}_{i n v}=\left[\begin{array}{ccccccccc}
-1 & 0 & 0 & -1 & 0 & 0 & -1 & 0 & 0 \\
0 & -1 & 0 & 0 & -1 & 0 & 0 & -1 & 0 \\
0 & 0 & -1 & 0 & 0 & -1 & 0 & 0 & -1
\end{array}\right]^{T} .
\end{aligned}
$$

The matrix $\boldsymbol{A}_{i n v}$ for the inverse kinematic task and after transformations has the following formula

$$
A_{\text {inv }}=\left[\begin{array}{ccccccccc}
a_{11}^{1} & a_{12}^{1} & b_{13}^{1} & 0 & 0 & 0 & 0 & 0 & 0 \\
a_{21}^{1} & a_{22}^{1} & b_{23}^{1} & 0 & 0 & 0 & 0 & 0 & 0 \\
a_{31}^{1} & a_{32}^{1} & b_{33}^{1} & 0 & 0 & 0 & 0 & 0 & 0 \\
0 & 0 & 0 & a_{11}^{2} & a_{12}^{2} & b_{13}^{2} & 0 & 0 & 0 \\
0 & 0 & 0 & a_{21}^{2} & a_{22}^{2} & b_{23}^{2} & 0 & 0 & 0 \\
0 & 0 & 0 & a_{31}^{2} & a_{32}^{2} & b_{34}^{2} & 0 & 0 & 0 \\
0 & 0 & 0 & 0 & 0 & 0 & a_{11}^{3} & a_{12}^{3} & b_{13}^{3} \\
0 & 0 & 0 & 0 & 0 & 0 & a_{21}^{3} & a_{22}^{3} & b_{23}^{3} \\
0 & 0 & 0 & 0 & 0 & 0 & a_{31}^{3} & a_{32}^{3} & b_{33}^{3}
\end{array}\right]
$$

where for $\quad j=1,2,3$

$$
\begin{aligned}
& b_{13}^{j}=\cos \gamma_{j} \sin \beta_{j}+\sin \gamma_{j} \cos \beta_{j} \sin \alpha_{j}, \\
& b_{23}^{j}=\sin \gamma_{j} \sin \beta_{j}-\cos \gamma_{j} \cos \beta_{j} \sin \alpha_{j}, \\
& b_{33}^{j}=\cos \alpha_{j} \cos \beta_{j} .
\end{aligned}
$$




\section{Analysis of singular positions of MT-3-UPU mechanism}

During their operation mechanisms may assume link positions in which they are kinematically indeterminate. Such mechanism positions are commonly referred to as singular. When a system is brought to a singular position it changes its kinematic and dynamic properties: the values of some parameters (forces, velocities and accelerations) become indeterminate or approach infinity (Tsai, 1999; Gronowicz, 2003; Bałchanowski, 2012; 2014).

In the direct and inverse kinematics problems of the mechanism under consideration singular configurations occur when matrices $\boldsymbol{A}_{\text {dir }}$ and $\boldsymbol{A}_{\text {inv }}$ from velocity Eq.(2.11) and Eq.(2.12) are singular (eg. Gronowicz, 2003; Bałchanowski, 2012), i.e., when

$$
\begin{aligned}
& \operatorname{det}\left(A_{\text {dir }}\right)=0, \\
& \operatorname{det}\left(\boldsymbol{A}_{\text {inv }}\right)=0 .
\end{aligned}
$$

After determinant Eq.(3.1) is determined and algebraic transformations are performed, singular positions for the direct kinematics problems will occur provided that the following equation is equal to zero

$$
\operatorname{det}\left(A_{\text {dir }}\right)=\left\{\begin{array}{l}
-q_{1}^{2} q_{2}^{2} q_{3}^{2} \cos \beta_{1} \cos \beta_{2} \cos \beta_{3} * \\
+\cos \alpha_{3} \cos \beta_{3}\left(\begin{array}{l}
\sin \beta_{2}\left(h_{1} \sin \beta_{1}+h_{2} \cos \beta_{1} \sin \alpha_{1}\right)+ \\
+\cos \beta_{2} \sin \alpha_{2}\left(h_{3} \sin \beta_{1}+h_{4} \cos \beta_{1} \sin \alpha_{1}\right)
\end{array}\right)+ \\
+\cos \alpha_{2} \cos \beta_{2}\left(\begin{array}{l}
\sin \beta_{1}\left(h_{5} \sin \beta_{3}+h_{6} \cos \beta_{3} \sin \alpha_{3}\right)+ \\
+\cos \beta_{1} \sin \alpha_{1}\left(h_{7} \sin \beta_{3}+h_{8} \cos \beta_{3} \sin \alpha_{3}\right)
\end{array}\right)+ \\
+\cos \alpha_{1} \cos \beta_{1}\left(\begin{array}{l}
\sin \beta_{3}\left(h_{9} \sin \beta_{2}+h_{10} \cos \beta_{2} \sin \alpha_{2}\right)+ \\
+\cos \beta_{3} \sin \alpha_{3}\left(h_{11} \sin \beta_{2}+h_{12} \cos \beta_{2} \sin \alpha_{2}\right)
\end{array}\right)
\end{array}\right)
$$

where $h_{i}=\left(\gamma_{1}, \gamma_{2}, \gamma_{3}\right)-$ constants being functions of angles $\gamma_{1}, \gamma_{2}, \gamma_{3}$.

The equation holds true when any of the elements of the product in Eq.(3.3) is equal to zero. This occurs in the cases

a)

b) $\quad \beta_{1}= \pm \pi / 2$ or $\beta_{2}= \pm \pi / 2$ or $\beta_{3}=\pi / 2$,

c) $\quad \alpha_{1}, \alpha_{2}, \alpha_{3}= \pm \pi / 2$,

d) $\quad \alpha_{1}, \alpha_{2}, \alpha_{3}, \beta_{1}, \beta_{2}, \beta_{3}=0$.

Condition a) means that joints $A$ and $P$ (or $B$ and $S$, or $C$ and $R$ ) are coincident. Condition b) occurs for the perpendicular arrangement of the links in universal joints $A, B, C$ (Figs 2 and 3). Condition c) means that branches $A P, B R, C S$ of the mechanism (Figs 2 and 3) are parallel to the base and condition c) means that branches $A P, B R, C S$ of the mechanism are perpendicular to the base. Figures $4 \mathrm{a}, 4 \mathrm{~b}, 4 \mathrm{c}$ and $4 \mathrm{~d}$ show the considered mechanism in its singular positions. 
a)

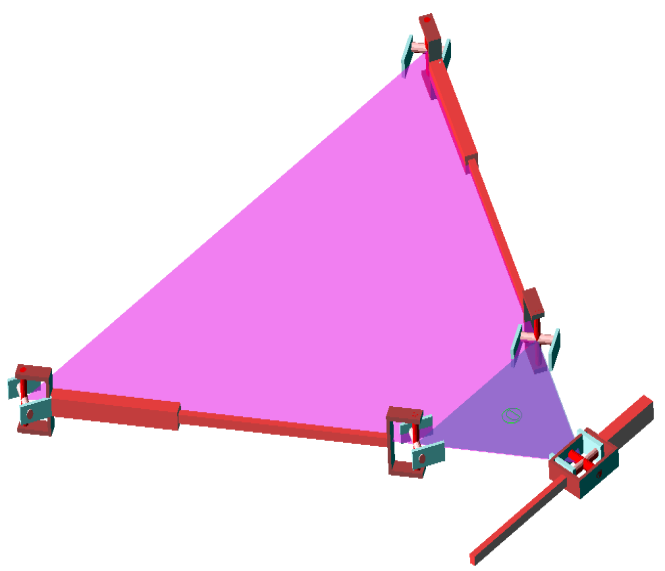

c)

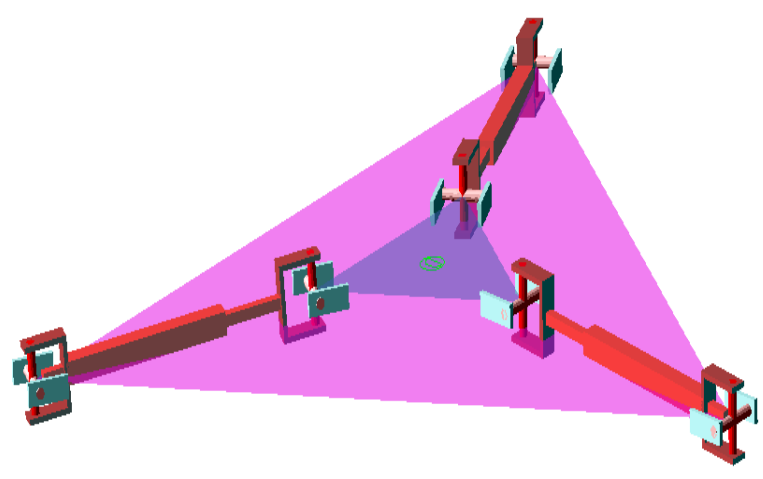

b)

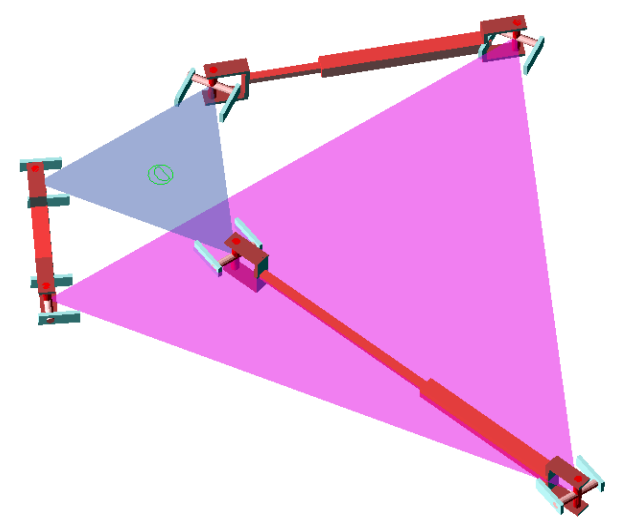

d)

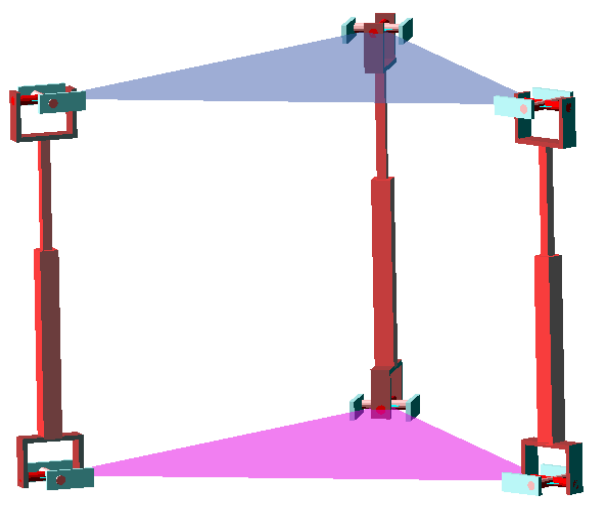

Fig.4. Examples of singular configurations of MT-3-UPU mechanism for direct and inverse kinematics problems: a) for $\left.q_{1}=0, \mathrm{~b}\right)$ for $\beta_{1}=\pi / 2$, c) for $\alpha_{i}=0(i=1,2,3)$, d) for $\alpha_{i}, \beta_{i}=0(i=1,2,3)$.

The singular positions for the inverse kinematics problems will occur provided that the following Eq.(3.2) describing the determinant is equal to zero

$$
\operatorname{det}\left(\boldsymbol{A}_{\text {inv }}\right)=q_{1}^{2} q_{2}^{2} q_{3}^{2} \cos \beta_{1} \cos \beta_{2} \cos \beta_{3} .
$$

Equation (3.4) holds true when any of the products is equal to zero

$$
q_{i}=0 \quad \text { or } \quad \beta_{i}= \pm \pi / 2, \quad \text { for } \quad i=1,2,3 \text {. }
$$

The next Figs $4 \mathrm{a}, 4 \mathrm{~b}$ show the mechanism in its singular positions for the inverse kinematic task.

\section{Conclusions}

In this paper, the kinematics characteristics of the 3-UPU parallel mechanism (with pure translational motion) are studied. The method of determination of mechanism configurations using the contour vector notation was presented in the paper.

The summary results of the kinematic analysis of the parallel mechanisms proved the existence of singular configurations both for the direct and inverse kinematic tasks. The occurrence of singular positions in the workspace of parallel mechanisms is a drawback. Therefore such analyses should not be neglected in 
the design process. If a mechanism of this kind is to be employed, it should be so designed as to prevent it from being brought to a singular position while performing tasks.

The next step after the singularity analysis of parallel robots, which determines the singular configurations, should be the analysis which predicts the mechanism behaviour near these configurations.

\section{Nomenclature}

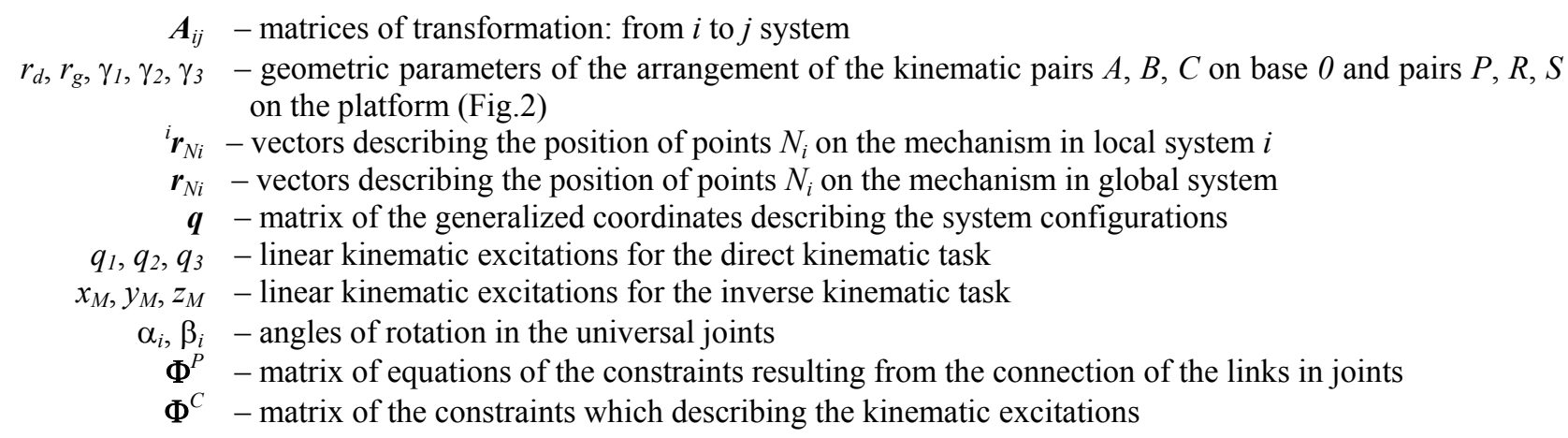

\section{References}

Bałchanowski J. (2014): Topology and analysis of the singularities of a parallel mechanism with three degrees of freedom. - Archives of Civil and Mechanical Engineering, vol.14, No.1, pp.80-87.

Bałchanowski J. (2008): Topology, geometry and kinematics of the selected translational parallel mechanisms (in Polish). -Mechanica Review, vol.67, No 10, pp.20-26.

Bałchanowski J. (2012): Direct and inverse kinematic of 3DOF parallel mechanism with singularity analysis. Advances in Mechanisms Design /eds. Jaroslav Beran. Dordrecht: Springer, cop. pp.89-96.

Bałchanowski J. and Gronowicz A. (2001): Topology and geometry of 3 dof parallel manipulators. - Eighth IFToMM International Symposium on Theory of Machines and Mechanisms. SYROM 2001, Bucharest, Romania.

Bałchanowski J., Gronowicz A., Miller S. and Twaróg W. (2001): Topology, kinematics and dynamics of the parallel manipulators. - Report no SPR 065/1999-2001. (in Polish), Wrocław University of Technology, Wrocław.

Chebii Ahmeg H., Affi Z. and Romdhane L.(2013): Kinematics, Workspace and Singularities Analysis of the 3-UPU Wrist Manipulator. - Design and Modeling of Mechanical System, LNME, Springer-Verlag, Berlin.

Di Gregorio R. and Parenti-Castelli V. (1999): Mobility Analysis of the 3-UPU Parallel Mechanism Assembled for a Pure Translational Motion. - Proc. of the 1999 IEEE/ASME Intern. Conference on Advanced Inteligent Mechatronics, Atlanta, USA.

Gogu G. (2008): Constraint singularities and the structural parameters of parallel robots. - Advances in Robot Kinematics: Analysis and Design, pp.21-28.

Gronowicz A. (2003): Basics of Analysis of Kinematic Systems (in Polish). - Press Office of the Wrocław University of Technology.

Guanfeng Liu, Yunjiang Lou and Zexiang Li (2003): Singularities of parallel manipulators: A geometric treatment. IEEE Transactions on Robotics and Automation, vol.19, No.4, 08/2003.

Kim D. and Chung W. (2003): kinematic condition analysis of three-DOF pure translational parallel manipulators. ASME Journal of Mechanical Design, 125(2), pp.323-331.

Merlet J.P. (2000): Parallel Robots. - London: Kluwer, Academic Publishers.

Peng Binbin, Li Zengming, Wu Kai and Sun Yu (2011): Kinematic characteristic of 3-UPU parallel manipulator in singularity and its application. - Int. J. Adv. Robotic Sy, vol.8, No.4, pp.54-64. 
Tsai L.W. (1996): Kinematics of a three-dof platform with three extensible limbs. Recent advances in robot kinematics. - Kluwer Academic Publishers, pp.401-410.

Tsai L.W. (1999): Robot Analysis. The Mechanics and Parallel Manipulators. - New York: John Wiley and Sons, Inc.

Walter D. and Husty M. (2011): Kinematic Analysis of the Tsai-3-UPU Parallel Manipulator using Algebraic Methods. - 13th World Congress in Mechanism and Machine Science, Guanajuato, Mexico.

Wolf A., Shoham M. and Park F.C. (2002): Investigation of singularities and self-motions of the 3-UPU robot. Advances in Robot Kinematics, pp.165-174.

Received: June 25, 2013

Revised: July 18, 2013 